This is an electronic reprint of the original article. This reprint may differ from the original in pagination and typographic detail.

Author(s): Särkkä, Olli; Nieminen, Tuukka; Suuriniemi, Saku; Kettunen, Lauri

Title: A Multi-Position Calibration Method for Consumer-Grade Accelerometers, Gyroscopes, and Magnetometers to Field Conditions

Year: $\quad 2017$

Version:

Please cite the original version:

Särkkä, O., Nieminen, T., Suuriniemi, S., \& Kettunen, L. (2017). A Multi-Position Calibration Method for Consumer-Grade Accelerometers, Gyroscopes, and Magnetometers to Field Conditions. IEEE Sensors Journal, 17(11), 3470-3481. https://doi.org/10.1109/JSEN.2017.2694488

All material supplied via JYX is protected by copyright and other intellectual property rights, and duplication or sale of all or part of any of the repository collections is not permitted, except that material may be duplicated by you for your research use or educational purposes in electronic or print form. You must obtain permission for any other use. Electronic or print copies may not be offered, whether for sale or otherwise to anyone who is not an authorised user. 


\title{
A multi-position calibration method for consumer-grade accelerometers, gyroscopes, and magnetometers to field conditions
}

\author{
Olli Särkkä, Tuukka Nieminen, Saku Suuriniemi, and Lauri Kettunen
}

\begin{abstract}
This paper presents a calibration method for consumer-grade accelerometers, gyroscopes, and magnetometers. Considering the calibration of consumer-grade sensors, it is essential that no specialized equipment is required to create reference signals. In addition, the less is required from the reference signals, the more suitable the method is on the field. In the proposed method, the novelty in the calibration of the gyroscopes lies in the exploitation of only the known net rotations between the positions in a multi-position calibration. For accelerometers and magnetometers, the innovation is that the direction of reference signals, the gravity and the magnetic field of the Earth, are estimated with calibration parameters. As a consequence, no precise absolute alignment of the sensors is needed in the calibration. The rotations need not be done about a constant axis. In the proposed method, the biases, scale factors, misalignments, and cross-coupling errors for all the sensors as well as hard iron and soft iron effect for magnetometers were modelled. In addition, the drift of the sensors during the calibration was estimated. As a result, all the sensors were calibrated at once to the same frame, exploiting only a cube and a jig and thus, the method is eligible in the field. To estimate the quality of the calibration results, $95 \%$ confidence intervals were calculated for the calibration parameters. Simulations were done to indicate that the calibration method is unbiased.
\end{abstract}

Index Terms-Multi-position calibration, inertial measurement unit (IMU), accelerometer, gyroscope, magnetometer, confidence interval.

\section{INTRODUCTION}

$\mathbf{T}$ HIS paper presents a calibration method for consumergrade $^{1}$ triaxial accelerometers, gyroscopes, and magnetometers. In general, the goal of the calibration is to estimate as many systematic sensor errors as possible. In practice, the raw output of the sensor is compared with a known input, the reference signal, to find the errors and adjust the sensor.

The calibration methods of accelerometers, gyroscopes, and magnetometers are often based on multi-position calibration [2], [3], where the fixed sensor assembly is kept in a number of different attitudes with respect to the reference signal. The reference signals can be either artificially created or associated to the Earth or both. For consumer-grade sensors, it is an advantage if the reference signals require no excessive equipment -such as a turn table or a Helmholtz coil. In

O. Särkkä, T. Nieminen, and S. Suuriniemi are with the Tampere University of Technology, Department of Electrical Engineering, P.O. Box 692, FI-33101 Tampere University of Technology, Finland (e-mail: olli.sarkka@gmail.com)

L. Kettunen is with the University of Jyvaskyla, Faculty of Information Technology, P.O. Box 35, FI-40014, University of Jyvaskyla, Finland

${ }^{1}$ For consumer-grade gyroscopes and accelerometers, the bias instability is greater than $0.5 \mathrm{mrad} / \mathrm{s}(100 \mathrm{deg} / \mathrm{h})$ and $0.03 \mathrm{~m} / \mathrm{s}^{2}$, respectively [1]. addition, it is often desirable that the calibration method is applicable on the field. In general, the less is required from the reference signals, the better the method is on the field.

A great number of calibration methods concerning the accelerometers, gyroscopes, and magnetometers can be found in the literature. Consumer-grade calibration methods [4]-[23] with the proposed method are summed up in Table I. In general, the calibration measurements, together with the sensor error models, determine the systematic sensor errors that can be detected.

The proposed gyroscope calibration method rely only on known net rotations, which can take place on the field, for example, by exploiting a cube and a jig. This approach differs from those shown in [4]-[8], [14], [15], see Table I. The method described in [9] also exploits known net rotations to estimate gyroscope scale factors and biases. In addition, the proposed method models misalignments and cross-coupling errors.

The accelerometers are calibrated with the aid of gravity. The direction of gravity need not be known (accurately). Instead, an initial guess indicating the positive direction of the gravity is enough. The calibration parameters and the direction of the gravity are estimated simultaneously by minimizing the residual of an overdetermined system of equations obtained by changing the position of the sensors with rotations. This is the novelty compared to the methods presented in [4]-[13], [16]. Again, net rotations need be known. The magnitude of the gravity need also be known to adjust the accelerometers to specific unit. (For estimation of the gravity, see for example [24].)

Reference [10] presents a nine-parameter calibration method with a necessary and sufficient condition to determine whether the calibration of accelerometers is possible or not. In our case a twelve-parameter sensor error model is exploited. The quality of the parameters is estimated with $95 \%$ confidence intervals. In addition, the estimated direction of gravity can be compared to (an externally) measured value.

Some calibration methods for magnetometers are presented in [16]-[20]. In [16] an auxiliary vector (for example a known attitude or calibrated sensor measurements), whose component in the direction of the magnetic field is constant, is needed to calibrate the magnetometers. References [17]-[20] exploit the magnitude of the magnetic field. The adjustment is done in a frame specified by the physical alignment of the sensor triad. The proposed method exploits the magnetic field of the Earth as reference signal enabling the adjustment to a 
TABLE I

COMPARISON OF CALIBRATION METHODS.

\begin{tabular}{|c|c|c|c|c|c|c|}
\hline Method & Sensors & Reference signals & $\begin{array}{l}\text { Calibration } \\
\text { parameters }\end{array}$ & $\begin{array}{l}\text { Calibration } \\
\text { measurements }\end{array}$ & $\begin{array}{l}\text { Lab only/ } \\
\text { Field }\end{array}$ & Notes \\
\hline$[4]$ & $\begin{array}{l}\text { acc } \\
\text { gyro }\end{array}$ & $\begin{array}{l}\text { constant position and zero } \\
\text { velocity of sensors }\end{array}$ & & manual motion & $\mathrm{F}$ & no equipment needed \\
\hline$[5]$ & $\begin{array}{l}\text { acc } \\
\text { gyro }\end{array}$ & $\begin{array}{l}\text { gravity } \\
\text { calibrated acc measurements }\end{array}$ & $\begin{array}{l}\mathrm{mis} \\
\mathrm{mis}\end{array}$ & $\begin{array}{l}\text { positions } \\
\text { arbitrary motion }\end{array}$ & $\mathrm{F}$ & no equipment needed \\
\hline$[6]$ & $\begin{array}{l}\text { acc } \\
\text { gyro }\end{array}$ & $\begin{array}{l}\text { gravity, } \\
\text { angular velocity }\end{array}$ & $\begin{array}{l}\mathrm{mis} \\
\mathrm{mis}\end{array}$ & $\begin{array}{l}\text { positions } \\
\text { rate table }\end{array}$ & $\mathrm{L}$ & $\begin{array}{l}\text { sensors not adjusted } \\
\text { to the same frame }\end{array}$ \\
\hline [7] & $\begin{array}{l}\text { acc } \\
\text { gyro }\end{array}$ & $\begin{array}{l}\text { gravity, centripetal } \\
\text { acceleration, angular velocity }\end{array}$ & $\begin{array}{l}\text { mis } \\
\text { mis, g-dep }\end{array}$ & $\begin{array}{l}\text { positions } \\
\text { rate table }\end{array}$ & $\mathrm{L}$ & \\
\hline$[8]$ & $\begin{array}{l}\text { acc } \\
\text { gyro }\end{array}$ & $\begin{array}{l}\text { gravity } \\
\text { angular velocity }\end{array}$ & $\begin{array}{l}\mathrm{mis} \\
\mathrm{mis}\end{array}$ & $\begin{array}{l}\text { positions } \\
\text { rate table }\end{array}$ & $\begin{array}{l}\text { F (acc) } \\
\text { L (gyro) }\end{array}$ & $\begin{array}{l}\text { sensors not adjusted } \\
\text { to the same frame }\end{array}$ \\
\hline [9] & $\begin{array}{l}\text { acc } \\
\text { gyro }\end{array}$ & $\begin{array}{l}\text { gravity } \\
\text { known rotation angles }\end{array}$ & & $\begin{array}{l}\text { positions, } \\
\text { manual rotations }\end{array}$ & $\mathrm{F}$ & \\
\hline$[10]$ & acc & gravity & mis & positions & $\mathrm{F}$ & \\
\hline$[11],[12]$ & acc & gravity & & arbitrary movements & $\mathrm{F}$ & \\
\hline$[13]$ & acc & magnitude of gravity & & unknown positions & $\mathrm{F}$ & \\
\hline$[14]$ & gyro & $\begin{array}{l}\text { calibrated acc and/or mag } \\
\text { measurements }\end{array}$ & mis & rotations & $\mathrm{F}$ & \\
\hline$[15]$ & gyro & $\begin{array}{l}\text { pseudo observations and acc } \\
\text { and mag measurements }\end{array}$ & & arbitrary movements & $\mathrm{F}$ & no equipment needed \\
\hline$[16]$ & $\begin{array}{l}\mathrm{acc} \\
\mathrm{mag}\end{array}$ & $\begin{array}{l}\text { known attitude or calibrated } \\
\text { sensor measurements }\end{array}$ & $\begin{array}{l}\text { mis } \\
\text { mis, hi, si }\end{array}$ & arbitrary rotation & $\mathrm{F}$ & \\
\hline [17], [18] & mag & magnetic field of the Earth & mis & $\begin{array}{l}\text { positions, } \\
\text { reference magnetometer }\end{array}$ & $\mathrm{L}$ & \\
\hline$[19]$ & mag & magnetic field of the Earth & mis, hi, si & rotations & $\mathrm{F}$ & \\
\hline$[20]$ & mag & magnetic field of the Earth & mis, hi, si & arbitrary motion & $\mathrm{F}$ & \\
\hline [21] & $\begin{array}{l}\text { acc } \\
\text { gyro } \\
\text { mag }\end{array}$ & $\begin{array}{l}\text { gravity } \\
\text { calibrated acc or mag meas. } \\
\text { magnetic field of the Earth }\end{array}$ & $\begin{array}{l}\text { mis } \\
\text { mis } \\
\text { mis, hi, si }\end{array}$ & $\begin{array}{l}\text { positions } \\
\text { arbitrary motion } \\
\text { positions }\end{array}$ & $\mathrm{F}$ & $\begin{array}{l}\text { additional measurements } \\
\text { needed to adjust the } \\
\text { sensors to the same frame }\end{array}$ \\
\hline$[22]$ & $\begin{array}{l}\text { acc } \\
\text { gyro } \\
\text { mag }\end{array}$ & $\begin{array}{l}\text { gravity } \\
\text { known positions } \\
\text { magnetic field of the Earth }\end{array}$ & $\begin{array}{l}\text { mis } \\
\text { mis } \\
\text { mis, hi, si }\end{array}$ & positions & $\mathrm{L}$ & \\
\hline$[23]$ & $\begin{array}{l}\text { acc } \\
\text { gyro } \\
\text { mag }\end{array}$ & $\begin{array}{l}\text { magnitude of gravity, } \\
\text { orientation differences, } \\
\text { magnitude of magnetic } \\
\text { field of the Earth }\end{array}$ & $\begin{array}{l}\text { mis } \\
\text { mis } \\
\text { mis, hi, si }\end{array}$ & $\begin{array}{l}\text { unknown positions } \\
\text { and rotations }\end{array}$ & $\mathrm{F}$ & $\begin{array}{l}\text { all the sensors } \\
\text { calibrated } \\
\text { to the same frame }\end{array}$ \\
\hline $\begin{array}{l}\text { Proposed } \\
\text { method }\end{array}$ & $\begin{array}{l}\text { acc } \\
\text { gyro } \\
\text { mag }\end{array}$ & $\begin{array}{l}\text { gravity } \\
\text { known net rotations } \\
\text { magnetic field of the Earth }\end{array}$ & $\begin{array}{l}\text { mis } \\
\text { mis } \\
\text { mis, hi, si }\end{array}$ & $\begin{array}{l}\text { positions } \\
\text { and rotations }\end{array}$ & $\mathrm{F}$ & $\begin{array}{l}\text { all the sensors } \\
\text { calibrated at once } \\
\text { to the same frame }\end{array}$ \\
\hline
\end{tabular}

Note: Calibration parameters in addition to scale-factors and biases.

Abbreviations: acc is accelerometer, gyro is gyroscope, and mag is magnetometer. Mis is misalignments (including cross-coupling errors), g-dep is g-depency of the gyroscopes, and hi and si are the hard iron and soft iron effect in calibration of the magnetometers. The letter L means calibration only in a laboratory and the letter $\mathrm{F}$ in-field calibration.

known sensors' frame, see Fig.1. As with the accelerometers, direction of the reference signal need not be known in advance. This is important since the explicit specification of magnetic field direction may not be possible without measurement equipments. However, to adjust the magnetometers to specific unit, the magnitude of the magnetic field need be known. (For measured data and estimations of the Earth magnetic field, see for example [25].)

The methods presented in [21]-[23] calibrates accelerometers, gyroscopes, and magnetometers, which is also the goal here. In our approach, the calibration of accelerometers, gyroscopes and magnetometers do not depend on each other. The sensors are calibrated all at once to the same frame exploiting only a cube and a jig. This is a step forward to the calibration techniques presented in [21]-[23].

To sum up, the novelties of the proposed method are:

- The known net rotations in multi-position calibration are exploited to calibrate the gyroscopes. A possible mistake in the calibration process can be easily detected.

- The calibration model for accelerometers and magne- tometers enables to estimate the direction of the reference signals simultaneously with the calibration parameters. A reference or measured value of magnitude is needed only for the gravity and magnetic field. In addition, there is a freedom in choosing the plane, where the calibration rotations take place, making the approach suitable for field conditions.

- The confidence intervals of calibration parameters provide us with a quality estimate.

This paper is organized as follows: A calibration system is presented in section II, and the calibration models and solution methods in section III and IV. The simulations and test calibrations are discussed in sections V and VI, and the results and conclusions are shown in sections VII and VIII, respectively. In addition, Appendix A and B present the derivation of the confidence intervals for the calibration parameters. 


\section{CALIBRATION SYSTEM}

In the proposed calibration method the accelerometers, gyroscopes, and magnetometers are kept in a number of different positions. The net rotations between the positions are known accurately and are used as a reference for gyroscopes. For accelerometers and magnetometers, the reference signals are the gravity and the magnetic field of the Earth, respectively. As consumer-grade sensors are considered, the angular velocity of the Earth is neglected. This is done to keep the calibration models simple. However, if more accurate sensors are considered, the angular velocity of the Earth should be modelled. In that case, it may also be reasonable to exploit a more accurate sensor error model, for example, to take the gdependency of gyroscopes into account. However, this makes the calibration of the gyroscopes dependent on the acceleration measurements.

This paper presents an example, where in total 24 different positions and 23 known net rotations between the positions are exploited, see Fig. 1. The positions are known with respect to calibration frame, see Fig. 2. The rotations between the positions can be done manually. The consecutive positions are chosen to differ $90 \mathrm{deg}$. from each other to keep the calibration simple to execute. However, the rotations between the position can be done freely (need not be done about a constant axis), provided that the measurement range of the gyroscopes does not exceed. In practice, the sensor assembly was attached inside a cube, which was rotated against a jig.

In all 24 positions, the sensors are kept stationary for about 5000 samples ( 5 seconds) to reduce the effect of the noise by averaging the acceleration and magnetic field measurements. The rotations between the positions cover both positive and negative measurement directions of the gyroscopes and correspondingly the positions cover both positive and negative measurement directions of accelerometers and magnetometers. The time to carry out the calibration measurements is about four minutes.

After the 24th position, the sensors can be rotated back to the first position to estimate the possible temporal instability of the sensors: when a sensor is in the same position again, the output of the sensor should be the same again in absence of drift. This holds for gyroscopes and for accelerometers and magnetometer in a constant field. For example, rise or decrease of the temperature of consumer-grade sensors can cause drift. Since two positions are exploited to observe drift, it is modeled with an affine function. The adjustment is done on raw sensor measurements.

In proposed method, all the sensors will be adjusted to sensors' frame, see Fig. 1. Care must be taken when assembling the measurement device inside the cube to keep the information about the axes of sensors' frame of the measurement device, if the measurement device is removed from the cube after the calibration.

\section{Calibration models}

In this section the sensor error model is first introduced and thereafter the calibration models are constructed for gyroscopes, accelerometers, and magnetometers. In the models, all
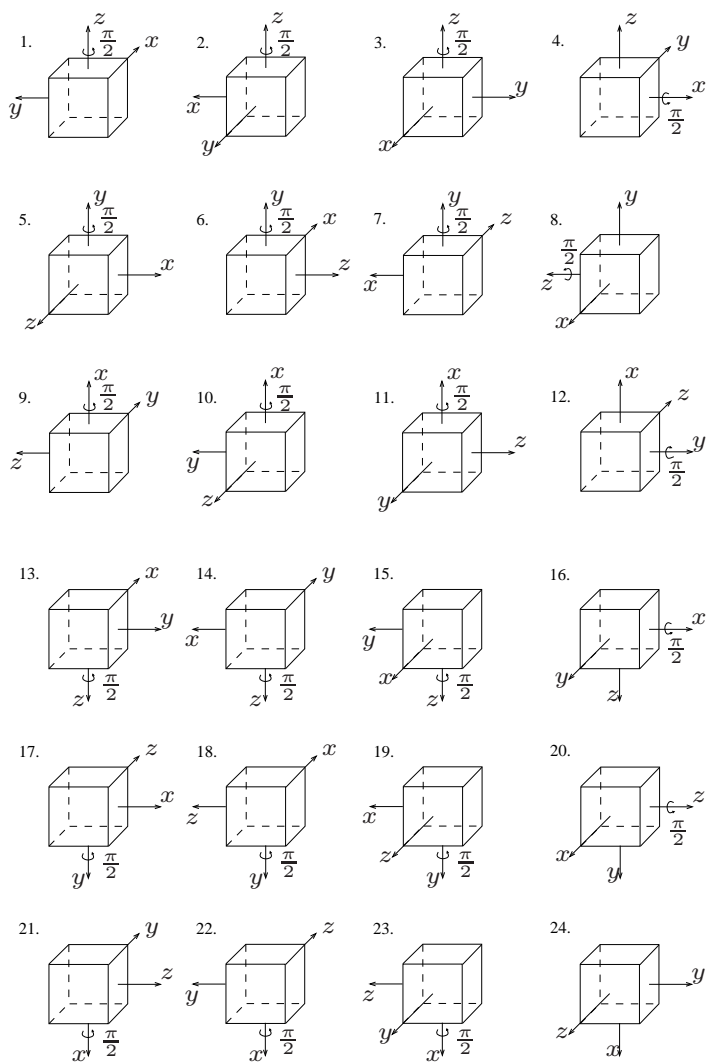

Fig. 1. 24 different positions, net rotations, and the axes of sensors' frame.

the reference signals are presented in the same frame, implying that all the sensors are calibrated to the same frame (sensors' frame), see Fig 1. In general, the different sensor triads should be adjusted to the same frame or alternatively the rotations between the different frames should be known, if the sensors are exploited together as for example accelerometers and gyroscopes are exploited in inertial navigation. If not adjusted or the rotations are not known, inter-sensors alignment errors occur.

\section{A. Sensor error model}

Two calibration parameters, a constant scale matrix $\mathbf{S}_{i} \in$ $\mathbb{R}^{3 \times 3}$ and bias $\mathbf{b}_{i} \in \mathbb{R}^{3}$, are estimated for accelerometers, gyroscopes, and magnetometers. The sensor error model [2] for each sensor is

$$
\tilde{\mathbf{f}}(t)=\mathbf{S}_{i} \mathbf{f}(t)+\mathbf{b}_{i}+\boldsymbol{\epsilon}(t),
$$

where the elements of $\tilde{\mathbf{f}}(t) \in \mathbb{R}^{3}$ are the raw outputs of the triaxial sensor, $\mathbf{f}(t) \in \mathbb{R}^{3}$ is a corresponding adjusted output, and $\boldsymbol{\epsilon}(t)$ is noise, $\boldsymbol{\epsilon}(t) \sim \mathcal{N}(0, \boldsymbol{\Sigma})$. In addition, $\mathbf{S}_{i}$ is nonsingular for nearly orthogonal triad of similar sensors.

To adjust the output of the sensors, it is enough to find $\mathbf{S}_{i}$ and $\mathbf{b}_{i}$. However, it is possible to choose different decompositions for $\mathbf{S}_{i}$, which lead to different interpretations of the calibration parameters. In this paper $\mathbf{S}_{i}$ is chosen to be $\mathbf{S}_{i}=\mathbf{D}_{i}\left(\mathbf{D}_{i}^{-1} \mathbf{S}_{i}\right)$, where $\mathbf{D}_{i}$ is a diagonal matrix. Its diagonal elements are the norms of the columns of $\mathbf{S}_{i}$, that is, the scale factors of the sensors. The matrix $\left(\mathbf{D}_{i}^{-1} \mathbf{S}_{i}\right)$ models the 
cross-coupling errors and misalignments of the sensors. This approach is unambiguous in the sense that there is no need to fix any of the measurement axis of the sensor triad. For magnetometers, $\left(\mathbf{D}_{B}^{-1} \mathbf{S}_{B}\right)$ and $\mathbf{b}_{B}$ model also hard iron and soft iron effect caused by magnetic material [20]. However, the sensor error model models the hard iron and soft iron effect properly, if magnetic material is "on board" that is, there is no translation and no rotation between magnetometers and the magnetic materials.

\section{B. Calibration model for gyroscopes}

The calibration parameters of the gyroscopes are estimated simultaneously with the attitude from the measured angular velocity data and the known net rotations. The position measurements between the calibration rotations are excluded. This is done to reduce the effect of the possible remaining drift on the results and to keep the problem reasonable size.

In general, the attitude can be solved from a differential equation, if the angular velocity over an interval in question and the attitude at a single time instant are known. The exploitation of the known net rotations within the interval enables the estimation of the calibration parameters.

The rotation from the reference attitude, i.e. the attitude, can be given as a quaternion-valued function $\mathbf{q}(t): \mathbb{R} \rightarrow \mathbb{R}^{4}$. If the initial attitude $\mathbf{q}\left(t_{0}\right)$ is known, the quaternions can be solved from an initial value problem with the differential equation [2]

$$
\dot{\mathbf{q}}(t)=\frac{1}{2} \mathbf{W}(\boldsymbol{\omega}(t)) \mathbf{q}(t) \quad \forall \quad t \in\left[t_{0}, t_{1}\right]
$$

where the elements of the matrix $\mathbf{W}$ are the given (adjusted) angular velocity $\boldsymbol{\omega}$ components

$$
\mathbf{W}(\boldsymbol{\omega}(t))=\left[\begin{array}{cccc}
0 & -\omega_{x}(t) & -\omega_{y}(t) & -\omega_{z}(t) \\
\omega_{x}(t) & 0 & \omega_{z}(t) & -\omega_{y}(t) \\
\omega_{y}(t) & -\omega_{z}(t) & 0 & \omega_{x}(t) \\
\omega_{z}(t) & \omega_{y}(t) & -\omega_{x}(t) & 0
\end{array}\right]
$$

and $\mathbf{q}$ is subject to the normality constraint

$$
\|\mathbf{q}(t)\|=1 \quad \forall \quad t \in\left[t_{0}, t_{1}\right] .
$$

Exploiting the sensor error model (1), the adjusted angular velocity $\boldsymbol{\omega}(t)$ in (2) is $\boldsymbol{\omega}(t)=\mathbf{S}_{\omega}^{-1}\left(\tilde{\boldsymbol{\omega}}(t)-\mathbf{b}_{\omega}\right)$. To approximate the solution of (2) with $\mathbf{S}_{\omega}^{-1}$ and $\mathbf{b}_{\omega}$ (for gyroscopes, instead of $\mathbf{S}_{\omega}$, it is more convenient to estimate matrix $\mathbf{S}_{\omega}^{-1}$, which is actually needed to adjust the gyroscopes), (2) need be given as a discretized system of equations. This can be done by applying a trapezoidal rule [26] to (2). The trapezoidal rule is symmetric for forward and backward process and this property is natural here, since the attitude for all time instances and the calibration parameters are estimated simultaneously. To require that the trapezoidal rule is satisfied approximately over the time interval $\left[t_{0}, t_{1}\right]$, the following residual equation is obtained

$$
\mathbf{r}_{1}(\mathbf{x})=\left[\begin{array}{c}
{\left[\frac{1}{4} h \mathbf{W}\left(\boldsymbol{\omega}_{1}\right)+\mathbf{I}\right] \mathbf{q}_{1}+\left[\frac{1}{4} h \mathbf{W}\left(\boldsymbol{\omega}_{2}\right)-\mathbf{I}\right] \mathbf{q}_{2}} \\
{\left[\frac{1}{4} h \mathbf{W}\left(\boldsymbol{\omega}_{2}\right)+\mathbf{I}\right] \mathbf{q}_{2}+\left[\frac{1}{4} h \mathbf{W}\left(\boldsymbol{\omega}_{3}\right)-\mathbf{I}\right] \mathbf{q}_{3}} \\
\vdots \\
{\left[\frac{1}{4} h \mathbf{W}\left(\boldsymbol{\omega}_{N-1}\right)+\mathbf{I}\right] \mathbf{q}_{N-1}+\left[\frac{1}{4} h \mathbf{W}\left(\boldsymbol{\omega}_{N}\right)-\mathbf{I}\right] \mathbf{q}_{N}}
\end{array}\right],
$$

where $\mathbf{r}_{1}$ is the residual, $\mathbf{x}=\left[\mathbf{q}_{1}^{T} \mathbf{q}_{2}^{T} \ldots \mathbf{q}_{N}^{T} \mathbf{s}_{\omega}^{T} \mathbf{b}_{\omega}^{T}\right]^{T}, \mathbf{I}$ is a $4 \times 4$ identity matrix, and $h=t_{i}-t_{i-1}$ is the step size. In $\mathbf{x}$, the vector $\mathbf{s}_{\omega} \in \mathbb{R}^{9}$ contains the columns of $\mathbf{S}_{\omega}^{-1}$. Equation (4) is non-linear, since in addition to the angular velocity measurements, the matrix $\mathbf{W}$ depends on unknown calibration parameters.

The trapezoid rule residual in expression (4) attains the zero value with a number of arguments, i.e. the corresponding equation is underdetermined with $4(N-1)$ residual equations and $4 N+12$ unknowns, where $N$ is the number of time indices. The trapezoid rule residual is therefore supplemented with a residual to account for the known net rotations. The initial attitude in the calibration coincides with a reference frame (the calibration frame in the next section), hence a quaternion corresponding the first attitude, is trivial. A forward ODE solution of (2) with pre-adjusted angular velocity data (in pre-adjustment, the gyroscopes are adjusted with the scale factors and biases taken from the data sheet of the sensor and estimated from static sensor measurements, respectively) approximates the quaternion at the end of the first rotation, and the exact end attitude can then be represented by the nearby quaternion of the correct branch ${ }^{2}$. Subsequent reference quaternions at the end of each net rotation are determined similarly and gathered into $\mathrm{x}^{\star}$. With this method the determination of reference quaternions can be automated.

All the quaternions to be estimated are included in $\mathbf{x}$, and a binary matrix $\mathbf{G}$ is constructed to associate the very first and then the last quaternion estimate of each rotation to the corresponding 24 reference values. The supplementary attitude residual can be expressed as

$$
\mathbf{r}_{2}(\mathbf{x})=\mathbf{G} \mathbf{x}-\mathbf{x}^{\star} .
$$

The proposed method integrates the measured angular velocity to find the attitude and the calibration parameters. Possible drift in measurement data can affect the estimated calibration parameters. However, the drift of the sensors is compensated as explained in section II. In addition, the output of the gyroscopes need to be integrated only over the each rotation period between the positions. The rotation time is kept short, about two seconds per rotation. If the integration time and thus the possible errors caused by the drift are to be reduced, it is also possible to do faster rotations, if the measurement range of gyroscopes permits. On the other hand, if the drift is random, the redundant rotations compensate the effect of the drift to calibration parameters.

In the next section, attitudes and the calibration parameters (vector $\mathbf{x}$ ) are estimated by minimizing the sum of squared residual norms $\left\|\mathbf{r}_{1}(\mathbf{x})\right\|$ and $\left\|\mathbf{r}_{2}(\mathbf{x})\right\|$ with different weight.

\section{Calibration models for accelerometers and magnetometers}

Accelerometers and magnetometers are calibrated independently, but the calibration method is the same for both sensor. The calibration parameters are estimated from the means of the reference signal (the gravity and the magnetic field of the Earth) measurements made in the 24 different positions. The method also estimates the direction of reference signals and thus they need be known only approximately in advance.

\footnotetext{
${ }^{2}$ The quaternions $\mathbf{q}$ and $-\mathbf{q}$ always correspond to the same attitude.
} 
However, the magnitudes of reference signals must be known to adjust the sensors to specific units.

To construct the calibration models for accelerometers and magnetometers, the rotation between two reference frames is expressed by a direction cosine matrix $\mathbf{C}(t): \mathbb{R} \rightarrow \mathbb{R}^{3 \times 3}$. The matrix $\mathbf{C}_{l}^{c}$ maps the references from local frame, where the reference signals are presented, to the calibration frame, where the calibration rotations, presented in Fig. 1, are specified. The matrix $\mathbf{C}_{c}^{s}\left(t_{k}\right)$ maps the references from calibration frame to the sensors' frame, whose axes are shown in Fig. 1. With this notation, the calibration models for triaxial accelerometer and magnetometer at each unkonwn position are

$$
\begin{aligned}
\tilde{\mathbf{g}}\left(t_{k}\right) & =\mathbf{S}_{a} \mathbf{C}_{c}^{s}\left(t_{k}\right) \mathbf{C}_{l}^{c} \mathbf{g}+\mathbf{b}_{a}+\boldsymbol{\epsilon}_{a} \\
\tilde{\mathbf{B}}\left(t_{k}\right) & =\mathbf{S}_{B} \mathbf{C}_{c}^{s}\left(t_{k}\right) \mathbf{C}_{l}^{c} \mathbf{B}+\mathbf{b}_{B}+\boldsymbol{\epsilon}_{B},
\end{aligned}
$$

respectively. On the left in (6) and (7) $\tilde{\mathbf{g}}$ and $\tilde{\mathbf{B}}$ are the means of the gravity and the magnetic field measurements and on the right $\mathbf{g}$ and $\mathbf{B}$ are the corresponding references. For the meaning of $\mathbf{S}_{i}, \mathbf{b}_{i}$, and $\boldsymbol{\epsilon}_{i}$, see eq. (1).

The matrix $\mathbf{C}_{l}^{c}$ can be given in terms of two inclination angles as

$$
\begin{aligned}
\mathbf{C}_{l}^{c} & =\left[\begin{array}{ccc}
1 & 0 & 0 \\
0 & \cos \alpha & \sin \alpha \\
0 & -\sin \alpha & \cos \alpha
\end{array}\right]\left[\begin{array}{ccc}
\cos \beta & 0 & -\sin \beta \\
0 & 1 & 0 \\
\sin \beta & 0 & \cos \beta
\end{array}\right] \\
& =\left[\begin{array}{ccc}
\cos \beta & 0 & -\sin \beta \\
\sin \alpha \sin \beta & \cos \alpha & \sin \alpha \cos \beta \\
\cos \alpha \sin \beta & -\sin \alpha & \cos \alpha \cos \beta
\end{array}\right] .
\end{aligned}
$$

If the calibration frame and the local frame coincide, the angles $\alpha$ and $\beta$ are zero and $\mathbf{C}_{l}^{c}$ is an identity matrix, see Fig. 2. This holds approximately for calibration of accelerometers considered in the results section VII. The local plane is chosen so that the reference in question is perpendicular to it.

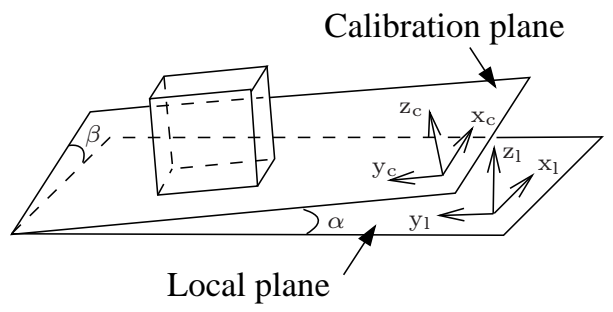

Fig. 2. The calibration plane and the local plane with corresponding frames.

The angles $\alpha$ and $\beta$ of the matrix $\mathbf{C}_{l}^{c}$ could be measured with other methods. However, the calibration is to be independent from additional measuring devices and thus the angles are estimated by the calibration routine. The matrix $\mathbf{C}_{c}^{s}\left(t_{k}\right)$ (the mapping between the calibration and sensors' frame) is known from Fig. 1, but it can also be determined exploiting the angular velocity measurements explained in section III-B. The first position is chosen to be the calibration frame. Hence, the calibration and sensors' frame are chosen to coincide at the first position in Fig. 1, implying that $\mathbf{C}_{c}^{s}\left(t_{1}\right)$ is the identity matrix.

As there are three equations per position, the total number of equations in calibration model is $3 \times 24=72$. The number of unknowns is 14, meaning that the system of equations is overdetermined.

During the calibration measurements it is assumed that reference signals are constant. This holds very accurately for gravity. Instead, the magnetic field of the Earth can change as a function of position. The changes in position can be eliminated by keeping the magnetometers in the same place in every position. This can be done installing the magnetometers in the centre of the cube and setting the cube to the same place after every rotation.

\section{SOLUTION METHODS}

The solution method is first given for the calibration model of the gyroscopes and thereafter for the accelerometers and magnetometers. In both cases, the residual norms of the calibration models are minimized, but different methods are applied.

\section{A. Gyroscopes}

To calibrate the gyroscopes, two residuals were derived, namely the expression (4) and the expression (5). The norms of the residuals can be minimized to find the attitudes and especially the calibration parameters. However, if the residual norms are to be satisfied with different accuracy, the weight between different residual norm can be set. Technically, this can be done with Tikhonov regularization.

A Tikhonov regularization problem [27] for residuals (4) and (5) can be given as

$$
\hat{\mathbf{x}}_{\lambda}=\underset{\mathbf{x}}{\operatorname{argmin}}\left\{\left\|\mathbf{r}_{1}(\mathbf{x})\right\|_{2}^{2}+\lambda\left\|\mathbf{r}_{2}(\mathbf{x})\right\|_{2}^{2}\right\} .
$$

where the real parameter $\lambda>0$ is called the regularization parameter. The regularization parameter weights the latter residual norm compared to the first residual norm. Accordingly, the greater the $\lambda$, the more the latter equation is weighted and thus the more the known positions are trusted. In addition, all the positions are weighted equally.

As the minimization problem (9) is non-linear, an iterative method is needed to find an approximative solution for it. In this case, Gauss-Newton [28] is applied. For this, the minimization problem (9) is rewritten in a least squares form

$$
\hat{\mathbf{x}}_{\lambda}=\underset{\mathbf{x}}{\operatorname{argmin}}\left\|\begin{array}{c}
\mathbf{r}_{1}(\mathbf{x}) \\
\lambda^{1 / 2} \mathbf{r}_{2}(\mathbf{x})
\end{array}\right\|_{2}^{2} .
$$

Gauss-Newton needs Jacobian matrix and an initial guess to operate. The Jacobian matrix $\mathbf{J}$ of (10) is

$$
\mathbf{J}=\left[\begin{array}{c}
\frac{d}{d \mathbf{x}} \mathbf{r}_{1}(\mathbf{x}) \\
\lambda^{1 / 2} \mathbf{G}
\end{array}\right]
$$

To choose the initial guess $\mathbf{x}_{0}$ for Gauss-Newton, the quaternions were solved as an initial value problem from (2) with initial value $\mathbf{q}_{0}=\left[\begin{array}{llll}1 & 0 & 0 & 0\end{array}\right]^{T}$ (for this choice the calibration and the sensor frame are parallel in the first (initial) position in Fig. 1 and pre-adjusted angular velocity $\boldsymbol{\omega}$. In pre-adjustment, the angular velocity measurements were adjusted with the scale factors given by the data sheet of a manufacturer [29] and the biases estimated from static sensor measurements. The solution of the initial value problem with the scale factors 
and biases were used as initial guess $\mathbf{x}_{0}$ for Gauss-Newton (the off-diagonal elements of $\mathbf{S}_{\omega}^{-1}$ were set to zero in initial guess $\mathbf{x}_{0}$ ). The more accurate initial guess for attitude can be obtained solving the initial value problem (2) over the each calibration rotation separately exploiting corresponding known position as an initial value.

The solution method does not guarantee that the normality condition (3) holds exactly. However, the norm of estimated quaternions differed from one in order of $10^{-5}$ including the rounding errors. However, if the difference is to be reduced, one possibility is to add residuals of pseudo-measurements $\mathbf{q}_{i}^{T} \mathbf{q}_{i}=1$ in latter residual norm in (9). This brings the norm of the quaternion closer to one.

1) Finding regularization parameter $\lambda$ : There are several methods to find the regularization parameter $\lambda$. Perhaps the most popular ones are quasioptimality criterion, generalized cross-validation, and L-curve criterion [27]. It turned out that, in this case, the L-curve criterion did not work. It also turned out that if $\lambda$ was changed within the interval $10^{-8}$ $10^{8}$ the corresponding changes in the calibration parameters were observed at third decimal. In addition, for small and great parameter values the calibration parameters saturated, that is, they did not change. Since the net rotation and thus the reference positions are known accurately, indicating a great parameter value, the $\lambda$ was chosen from the greater saturation point. In practice, the $\lambda$ was sought by increasing it and calculating the calibration parameters until they saturated. For this technique the parameter value $\lambda=10^{-1}$ was found and used to calculate the calibration parameters.

\section{B. Accelerometers and magnetometers}

The calibration parameters of the accelerometers and the magnetometers are estimated from the calibration models (6) and (7), respectively. Since the calibration models are nonlinear, an iterative method is needed to find the calibration parameters. If information about the reliability of the measurements is available, a weight matrix can be exploited to take the unreliability of the calibration measurements into account. The measurements of better quality will be weighted more than the measurements of lower quality.

For presentational reasons, let us write both calibration models (6) and (7) in a form $\mathbf{y}=\mathbf{h}(\mathbf{x})$. Its residual $\mathbf{p}(\mathbf{x})$ is

$$
\mathbf{p}(\mathbf{x})=\mathbf{h}(\mathbf{x})-\mathbf{y}
$$

To find an estimate for the calibration parameters $\mathbf{x}$, it is possible to look for a solution for a non-linear generalized least squares problem

$$
\hat{\mathbf{x}}=\underset{\mathbf{x}}{\operatorname{argmin}}\left\{\mathbf{p}(\mathbf{x})^{T} \mathbf{W} \mathbf{p}(\mathbf{x})\right\},
$$

where $\mathbf{W}$ is the weight matrix. The algorithm to solve the non-linear generalized least squares problem goes as follows [30]:

1) Choose an initial guess $x_{0}$ and a suitable stopping criterion $\delta$. Set $k=0$.

2) Compute the Jacobian matrix $\mathbf{J}_{k}=\frac{d}{d \mathbf{x}} \mathbf{h}\left(\mathbf{x}_{k}\right)$
3) Compute $\mathbf{x}_{k+1}=\mathbf{x}_{k}-\Delta \mathbf{x}_{k}$, where $\Delta \mathbf{x}_{k}$ is the linear solution of $\left(\mathbf{J}_{k}^{T} \mathbf{W} \mathbf{J}_{k}\right) \Delta \mathbf{x}_{k}=\mathbf{J}_{k}^{T} \mathbf{W} \mathbf{p}\left(\mathbf{x}_{k}\right)$.

4) If stopping criterion $\left\|\mathbf{x}_{k+1}-\mathbf{x}_{k}\right\| \leq \delta$ is not met, set $k=k+1$ and continue from step 2). Otherwise discontinue the iteration.

In the generalized least squares problem, the weight matrix $\mathbf{W}$ is the inverse of the variance-covariance matrix of the measurements $\boldsymbol{\Sigma}$. In general, $\boldsymbol{\Sigma}$ is not usually known for sure, but in some case it can be approximated. In this case, $3 \times 3$ diagonal blocks of $\boldsymbol{\Sigma}$ concerning three sets of measurements in each position is approximated with their sample covariance matrix multiplied by $1 / n$, where $n$ is the number of samples in each static sensor measurement. The multiplication by $1 / n$ is an effect of the exploitation of the sample means in the calibration, and is explained more detailed in Appendix A. The measurements concerning different positions are assumed independent, meaning that the off-diagonal blocks of $\boldsymbol{\Sigma}$ are zeroes. The method is akin to maximum likelihood estimation.

The initial guess was chosen by exploiting data sheets of the sensors and the knowledge that the directions of the measurement axes of the sensors are close to the axes of sensors' frame: The biases and the scale factors given by data sheets were used for the initial guess of $\mathbf{b}_{i}$ and diagonal elements of $\mathbf{S}_{i}$. The off-diagonal elements of $\mathbf{S}_{i}$ and the angles $\alpha$ and $\beta$ were set to zero.

If the direction of the sensors' axes are not close to the axes of sensors frame, the off-diagonal elements of $\mathbf{S}_{i}$ are not approximately zero. If the angles $\alpha$ and $\beta$ can be estimated roughly with some other method, the problem becomes linear. The solution of linear estimation problem can be exploited for the initial guess of the non-linear minimization problem.

\section{Simulations}

Actual sensor measurements cannot be exploited to show if the proposed calibration method is unbiased or not, since the true calibration parameters are not known. To take a stand on the unbiasedness of the method, simulations were done.

\section{A. Simulation of gyroscope measurements}

To test the unbiasedness of the calibration method of the gyroscopes, the angular velocity data of calibration rotations was generated. Each rotation was generated exploiting Rodrigues' rotation formula. The rotation axis was made to change its direction during the rotation to make the generated angular velocity more realistic. The corresponding angular velocity measurements were created exploiting the sensor error model (1) with the generated angular velocity and the known calibration parameters (shown in the Table VI). The noise in sensor error model was chosen to be normally distributed with a variance of the order of the pre-adjusted measurements $\left(4 \times 10^{-4}(\mathrm{rad} / \mathrm{s})^{2}\right)$. With this setup, the simulation was repeated 100 times with different noise realizations.

A separate simulation was done to estimate the $95 \%$ confidence intervals of the calibration parameters for gyroscopes. For this the angular velocity data was created and it was chosen to be sine and cosine functions. The corresponding angular velocity measurements were created exploiting the sensor error 
model (1) with the angular velocity data and the known calibration parameters. The attitude was estimated from the adjusted angular velocity measurements (the adjustment was done by exploiting the estimated calibration parameters) with the known initial and final attitudes. The confidence intervals were calculated for the estimated calibration parameters with 100 different noise realizations. The simulation results are shown in section VII-C.

\section{B. Simulation of accelerometer and magnetometer measure- ments}

To study the calibration method of accelerometers and magnetometer, measurement data was created. The calibration models (6) and (7) were used with the magnitudes of references $\|\mathbf{g}\|=9.80665 \mathrm{~m} / \mathrm{s}^{2}$ and $\|\mathbf{B}\|=51000 \mathrm{nT}$ and the known calibration parameters (presented in the Table VI). The angles $\alpha$ and $\beta$ were set 15 and $10 \mathrm{deg}$. and the noise was normally distributed with variances of the order of the real measurements $\left(2 \times 10^{-8} \mathrm{~V}^{2}\right.$ for accelerometers and $6 \times 10^{-8}$ $\mathrm{V}^{2}$ for magnetometers). The simulations were repeated 1000 times with different noise realizations. The results are shown in section VII-C.

\section{TESTS}

The proposed calibration method was tested with four custom-built measurement units (size $17 \mathrm{~cm}$ by $2 \mathrm{~cm}$ and mass $13 \mathrm{~g}$ without battery), that contained a triaxial $\pm 16 \mathrm{~g}$ accelerometer [31], three $\pm 105 \mathrm{rad} / \mathrm{s}$ gyroscopes [29], and a triaxial $\pm 0.6 \mathrm{mT}$ magnetometer [32]. The analog signals of the sensors were converted to digital form with a 24-bit ADconverter at $1 \mathrm{kHz}$ sampling frequency. The aluminium cube, exploited in calibration measurements, is shown in Fig. 3 with four measurement units. The same measurement unit was used in [33] to analyse javelin throwing mechanics.

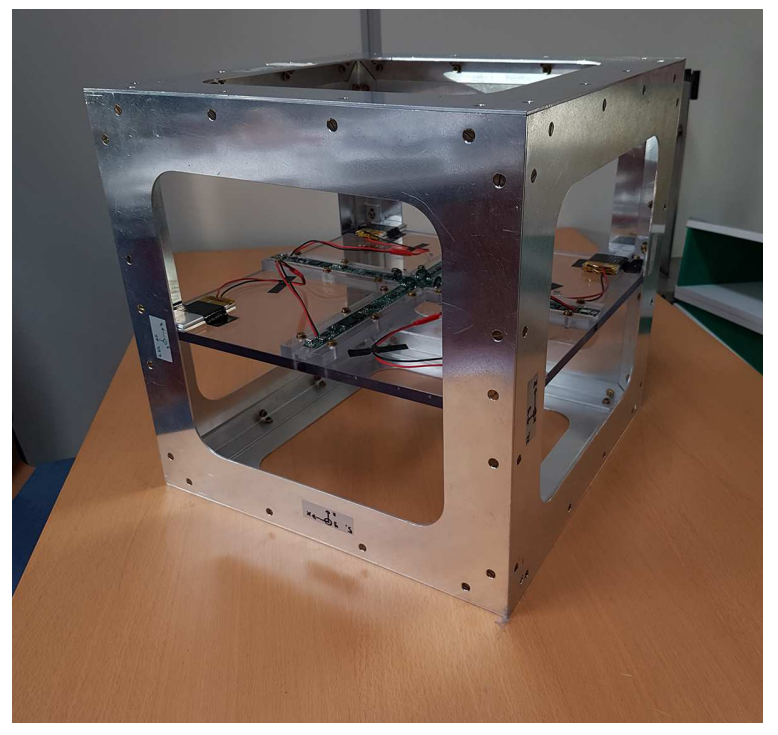

Fig. 3. The cube with four measurement units.

Since the estimated calibration parameters do not alone give information whether the calibration was successful or not, it is important to verify and produce information about the quality of the calibration parameters. In this paper this is done as follows:

- The estimated angles $\alpha$ and $\beta$ (the direction of the gravity) in calibration of accelerometer were used as a criterion for a successful calibration, since they can be measured with other methods and compared to the estimated angles. In addition, if more than one measurement unit is calibrated at the same time, the angles of different measurement units are the same (if there are no unmodelled sensor errors present) in successful calibration.

- Confidence intervals for calibration parameters of accelerometers, magnetometers, and gyroscopes were also calculated. Especially, the confidence intervals of biases give information about the quality of the calibration parameters.

The calibration method was tested on a horizontal plane and on an inclined plane. In addition, the robustness of the method was studied.

\section{RESULTS}

In this section, the calibration and simulation results are given and discussed.

\section{A. Calibration results of a horizontal plane}

An example of calibration parameters of the gyroscopes, accelerometers, and magnetometers (measurement unit 1 in Table IV) are given in Table II with $95 \%$ confidence intervals. The calibration parameters were estimated from calibration rotations made manually on a plane, which was set horizontal with spirit level with $\pm 0.2 \mathrm{deg}$. accuracy. In the calculation, the calibration parameters converged (towards optimal solution) in less than 8 iterations. The magnitudes of the reference signals were $\|\mathbf{g}\|=9.80665 \mathrm{~m} / \mathrm{s}^{2}$ and $\|\mathbf{B}\|=51000$ nT. The magnitude of $\|\mathbf{B}\|$ was taken from [34]. Derivation of the confidence intervals is presented in Appendix A for accelerometers and magnetometers and in Appendix B for gyroscopes.

In the calibration of the gyroscopes, the calibration parameters $\mathbf{S}_{\omega}^{-1}$ and $\mathbf{b}_{\omega}$ were first estimated from the pre-adjusted angular velocity measurements ${ }^{3}$, and the known positions. Thereafter, $\mathbf{D}_{\omega}$ and $\mathbf{D}_{\omega}^{-1} \mathbf{S}_{\omega}$ were calculated from $\mathbf{S}_{\omega}^{-1}$. Due to the pre-adjustment, $\mathbf{D}_{\omega}$ can be interpreted as scale factor error and $\mathbf{D}_{\omega}^{-1} \mathbf{S}_{\omega}$ as misalignments and cross-coupling errors of the pre-adjustment. For accelerometers and magnetometers, the calibration parameters $\mathbf{D}_{i}, \mathbf{D}_{i}^{-1} \mathbf{S}_{i}$, and $\mathbf{b}_{i}$ were estimated from raw sensor measurements.

The confidence intervals of the calibration parameters of gyroscopes were not calculated from the calibration measurements, since this led to a problem of prohibitive size. For this reason a separate test measurement, in which the gyroscopes were randomly rotated for about 5 seconds, was done to estimate the confidence intervals. The attitude was estimated

\footnotetext{
${ }^{3}$ Pre-adjustment is done to find an initial value of the attitudes for a nonlinear minimization problem, see section IV-A.
} 
TABLE II

A NUMERICAL EXAMPLE OF THE CALIBRATION PARAMETERS OF THE GYROSCOPES, ACCELEROMETERS, AND MAGNETOMETERS WITH $95 \%$ CONFIDENCE INTERVALS ESTIMATED FROM CALIBRATION MEASUREMENTS DONE ON A HORIZONTAL PLANE.

\begin{tabular}{r|rrr|r}
$\operatorname{diag}\left(\mathbf{D}_{\omega}\right)$ & \multicolumn{3}{|c|}{$\mathbf{D}_{\omega}^{-1} \mathbf{S}_{\omega}$} & $\mathbf{b}_{\omega}(\mathrm{mrad} / \mathrm{s})$ \\
\hline $0.9392 \pm 0.0048$ & $1.0000 \pm 0.006$ & $0.0006 \pm 0.011$ & $-0.0009 \pm 0.0037$ & $0.5603 \pm 1.0$ \\
$0.9404 \pm 0.0036$ & $-0.0006 \pm 0.015$ & $1.0000 \pm 0.004$ & $0.0009 \pm 0.0082$ & $-0.3923 \pm 0.8$ \\
$0.9837 \pm 0.0097$ & $0.0000 \pm 0.007$ & $-0.0018 \pm 0.005$ & $1.0000 \pm 0.0098$ & $-1.6733 \pm 1.6$ \\
\hline $\operatorname{diag}\left(\mathbf{D}_{a}\right)\left(\mu \mathrm{V} / \mathrm{m} / \mathrm{s}^{2}\right)$ & \multicolumn{3}{|c|}{} & $\mathbf{D}_{a}^{-1} \mathbf{S}_{a}$ \\
\hline $6230.3 \pm 0.18$ & $0.9999 \pm 0.28 \mathrm{e}^{-4}$ & $0.0326 \pm 0.28 \mathrm{e}^{-4}$ & $0.0002 \pm 0.29 \mathrm{e}^{-4}$ & $1617.9 \pm 0.0010$ \\
$6269.9 \pm 0.15$ & $-0.0123 \pm 0.23 \mathrm{e}^{-4}$ & $0.9995 \pm 0.24 \mathrm{e}^{-4}$ & $-0.0024 \pm 0.25 \mathrm{e}^{-4}$ & $1622.6 \pm 0.0009$ \\
$6032.3 \pm 0.31$ & $-0.0024 \pm 0.50 \mathrm{e}^{-4}$ & $0.0021 \pm 0.49 \mathrm{e}^{-4}$ & $0.9999 \pm 0.50 \mathrm{e}^{-4}$ & $1608.0 \pm 0.0017$ \\
\hline $\operatorname{siag}\left(\mathbf{D}_{B}\right)(\mathrm{V} / \mathrm{T})$ & & $\mathbf{D}_{B}^{-1} \mathbf{S}_{B}$ & $\mathbf{b}_{B}(\mathrm{mV})$ \\
\hline $2749.5 \pm 0.067$ & $1.0000 \pm 0.25 \mathrm{e}^{-4}$ & $-0.0163 \pm 0.24 \mathrm{e}^{-4}$ & $0.0630 \pm 0.24 \mathrm{e}^{-4}$ & $2588.0 \pm 0.0020$ \\
$2358.3 \pm 0.062$ & $-0.0035 \pm 0.27 \mathrm{e}^{-4}$ & $0.9998 \pm 0.27 \mathrm{e}^{-4}$ & $-0.0044 \pm 0.27 \mathrm{e}^{-4}$ & $2536.8 \pm 0.0019$ \\
$2244.4 \pm 0.058$ & $0.0050 \pm 0.26 \mathrm{e}^{-4}$ & $0.0032 \pm 0.25 \mathrm{e}^{-4}$ & $0.9970 \pm 0.26 \mathrm{e}^{-4}$ & $2544.5 \pm 0.0017$ \\
\hline
\end{tabular}

from adjusted angular velocity and the known initial and final attitudes. The attitude estimation is akin to calibration method of the gyroscopes, see section IV-A, with a difference that the calibration parameters are now known. The estimated attitude and the calibration parameters were used to estimate the confidence interval. This approach exaggerates the magnitude of the confidence intervals, since instead of 24 known positions only two reference attitudes were exploited. For accelerometers and magnetometers, the confidence interval of the calibration parameters were estimated from the calibration measurements.

1) Erroneous positions and robustness of the method: The expected exact end attitude of each rotation is known from Fig. 1, but it can be determined from forward ODE solution of (2). If the estimated end quaternion is not near to the reference quaternion (in calibrations, the estimated elements of the quaternions differed in maximum 0.05 from the exact elements and less than 0.1 difference in all elements represents the same nearby quaternion), there is a reason to believe that the user has done a mistake during the calibration rotations. However, if over 0.1 differences are found and the rotations after erroneous rotation do not follow the rotations presented in Fig. 1, but are known, the calibration is possible. Even if the rotations cannot be recovered after the erroneous rotation, the rotations before the mistake can be exploited in calibration.

To demonstrate this and robustness of the proposed calibration method, the calibration parameters, shown in the Table III, were recalculated for the gyroscopes, accelerometers, and magnetometers by excluding the positions 21-24 in Fig. 1. A comparison between calibration parameters with the excluded positions and the parameters calculated by exploiting all the positions reveals that the differences in the calibration parameters are small. Thus, the method can be considered robust and the possible error made by the user, especially towards the end of the calibration rotations, does not ruin the calibration.

2) The angles $\alpha$ and $\beta$ and the biases of the sensors: Since the calibration rotations were done on a plane that was set horizontal with accuracy of $\pm 0.2 \mathrm{deg}$., the estimated angles $\alpha$ and $\beta$ in the calibration of the accelerometers should not deviate from zero more than $\pm 0.2 \mathrm{deg}$. in a successful calibration. The angles with $95 \%$ confidence interval are shown in Table IV for four different measurement units. The $95 \%$ confidence interval means that there is $95 \%$ probability that the confidence interval includes the true value (not known, but estimated).

The estimated angles $\alpha$ and $\beta$ are close to zero degrees and inside the given interval $\pm 0.2 \mathrm{deg}$. The $95 \%$ confidence intervals are also well inside the interval $\pm 0.2 \mathrm{deg}$. However, the estimated angles with $95 \%$ confidence intervals between different measurement devices do not overlap. This can be a result from the fact that there are unmodelled sensor errors present causing error to the estimated angles and/or the estimated covariance matrices used in calculations of calibration parameters and confidence intervals differ from the true covariance matrices. However, since the angles are of the same order, the calibration of the accelerometers can be considered successful.

Table IV shows also the $95 \%$ confidence intervals for the biases of the accelerometers, magnetometers, and gyroscopes for all four measurement units. For accelerometers and magnetometers the confidence intervals of the biases are given as voltages and SI units. The confidence intervals of the biases of accelerometers in different measurement units are close to each other. However, in every measurement unit, the confidence interval of the bias of the $\mathrm{z}$-axis accelerometer is about double compared with other two axes. This may be because the specifications [31] for z-axis accelerometer were different from other two axes. These results indicate that after the adjustment the accuracy of the $x$ - and $y$-axis of accelerometer is greater than the z-axis. The confidence intervals of the biases of the magnetometers are close to each other and no significant or systematic differences occur between different measurement units. The same holds also for the gyroscopes.

\section{B. Calibration results of inclined plane}

To demonstrate that the calibration parameters can be estimated also from measurements made on an inclined plane, the calibration was repeated for measurement unit 1 on a calibration plane, which deviated from horizontal with the angles $\alpha=14.9 \mathrm{deg}$. and $\beta=10.1 \mathrm{deg}$. with accuracy \pm 0.3 deg., see Fig. 2. The estimated calibration parameters for the gyroscopes, accelerometers, and magnetometers with $95 \%$ confidence interval are shown in the Table V. The differences between the parameters estimated from calibration measurements made on the inclined plane and on the horizontal plane include run-to-run biases. 
TABLE III

THE ESTIMATED CALIBRATION PARAMETERS FOR THE GYROSCOPES, ACCELEROMETERS, AND MAGNETOMETERS WITH 95 \% CONFIDENCE INTERVAL, WHEN THE POSITIONS 21-24 IN FIG. 1 ARE EXCLUDED IN CALIBRATION.

\begin{tabular}{r|rrr|r}
$\operatorname{diag}\left(\mathbf{D}_{\omega}\right)$ & \multicolumn{3}{|c|}{$\mathbf{D}_{\omega}^{-1} \mathbf{S}_{\omega}$} & $\mathbf{b}_{\omega}(\mathrm{mrad} / \mathrm{s})$ \\
\hline $0.9409 \pm 0.005$ & $1.0000 \pm 0.005$ & $0.0005 \pm 0.020$ & $-0.0007 \pm 0.004$ & $0.1553 \pm 1.1$ \\
$0.9404 \pm 0.004$ & $-0.0008 \pm 0.028$ & $1.0000 \pm 0.004$ & $0.0020 \pm 0.012$ & $-0.2708 \pm 0.9$ \\
$0.9836 \pm 0.018$ & $0.0000 \pm 0.008$ & $-0.0020 \pm 0.007$ & $1.0000 \pm 0.018$ & $-1.7055 \pm 2.8$ \\
\hline $\operatorname{diag}\left(\mathbf{D}_{a}\right)\left(\mu \mathrm{V} / \mathrm{m} / \mathrm{s}^{2}\right)$ & $\mathbf{D}_{a}^{-1} \mathbf{S}_{a}$ & $\mathbf{b}_{a}(\mathrm{mV})$ \\
\hline $6228.4 \pm 0.28$ & $0.9999 \pm 0.45 \mathrm{e}^{-4}$ & $0.0326 \pm 0.28 \mathrm{e}^{-4}$ & $0.0002 \pm 0.29 \mathrm{e}^{-4}$ & $1617.9 \pm 0.0013$ \\
$6269.9 \pm 0.15$ & $-0.0120 \pm 0.38 \mathrm{e}^{-4}$ & $0.9995 \pm 0.24 \mathrm{e}^{-4}$ & $-0.0024 \pm 0.25 \mathrm{e}^{-4}$ & $1622.5 \pm 0.0011$ \\
$6032.3 \pm 0.31$ & $-0.0046 \pm 0.78 \mathrm{e}^{-4}$ & $0.0020 \pm 0.49 \mathrm{e}^{-4}$ & $1.0000 \pm 0.50 \mathrm{e}^{-4}$ & $1608.0 \pm 0.0021$ \\
\hline $\left.\operatorname{sing}\left(\mathbf{D}_{B}\right)(\mathrm{V} / \mathrm{T})\right)$ & \multicolumn{3}{|c|}{} & $\mathbf{b}_{B}(\mathrm{mV})$ \\
\hline $2740.1 \pm 0.095$ & $1.0000 \pm 0.35 \mathrm{e}_{B}^{-4}$ & $-0.0162 \pm 0.24 \mathrm{e}^{-4}$ & $0.0632 \pm 0.25 \mathrm{e}^{-4}$ & $2587.8 \pm 0.0024$ \\
$2359.6 \pm 0.062$ & $-0.0027 \pm 0.40 \mathrm{e}^{-4}$ & $0.9998 \pm 0.27 \mathrm{e}^{-4}$ & $-0.0042 \pm 0.27 \mathrm{e}^{-4}$ & $2536.8 \pm 0.0023$ \\
$2245.2 \pm 0.058$ & $0.0052 \pm 0.37 \mathrm{e}^{-4}$ & $0.0032 \pm 0.25 \mathrm{e}^{-4}$ & $0.9970 \pm 0.26 \mathrm{e}^{-4}$ & $2544.5 \pm 0.0020$ \\
\hline
\end{tabular}

TABLE IV

THE ESTIMATED ANGLES WITH $95 \%$ CONFIDENCE INTERVAL AND $95 \%$ CONFIDENCE INTERVAL FOR BIASES OF THE ACCELEROMETERS, MAGNETOMETERS, AND GYROSCOPES.

\begin{tabular}{rrrrr} 
Measurement unit & 1. & 2. & 3. & 4. \\
\hline$\alpha$ (deg.) & $0.0374 \pm 0.0010$ & $0.0417 \pm 0.0010$ & $0.0404 \pm 0.0009$ & $0.0346 \pm 0.0009$ \\
$\beta$ (deg.) & $0.0295 \pm 0.0010$ & $0.0197 \pm 0.0009$ & $0.0218 \pm 0.0009$ & $0.0249 \pm 0.0009$ \\
\hline acc $b_{x}(\mu \mathrm{V}),\left(\mathrm{mm} / \mathrm{s}^{2}\right)$ & $\pm 0.99, \pm 0.16$ & $\pm 0.92, \pm 0.15$ & $\pm 0.92, \pm 0.15$ & $\pm 0.88, \pm 0.14$ \\
acc $b_{y}(\mu \mathrm{V}),\left(\mathrm{mm} / \mathrm{s}^{2}\right)$ & $\pm 0.85, \pm 0.14$ & $\pm 0.85, \pm 0.14$ & $\pm 0.87, \pm 0.14$ & $\pm 0.84, \pm 0.14$ \\
acc $b_{z}(\mu \mathrm{V}),\left(\mathrm{mm} / \mathrm{s}^{2}\right)$ & $\pm 1.7, \pm 0.28$ & $\pm 1.7, \pm 0.28$ & $\pm 1.7, \pm 0.28$ & $\pm 1.6, \pm 0.26$ \\
\hline $\mathrm{mag} b_{x}(\mu \mathrm{V}),(\mathrm{nT})$ & $\pm 2.0, \pm 0.71$ & $\pm 1.6, \pm 0.58$ & $\pm 1.6, \pm 0.56$ & $\pm 1.5, \pm 0.56$ \\
$\operatorname{mag} b_{y}(\mu \mathrm{V}),(\mathrm{nT})$ & $\pm 1.9, \pm 0.79$ & $\pm 1.6, \pm 0.66$ & $\pm 1.6, \pm 0.67$ & $\pm 1.6, \pm 0.69$ \\
$\mathrm{mag} b_{z}(\mu \mathrm{V}),(\mathrm{nT})$ & $\pm 1.7, \pm 0.74$ & $\pm 1.5, \pm 0.65$ & $\pm 1.5, \pm 0.64$ & $\pm 1.5, \pm 0.65$ \\
\hline gyro $b_{x}(\mathrm{mrad} / \mathrm{s})$ & \pm 0.97 & \pm 0.65 & \pm 1.1 & \pm 0.95 \\
gyro $b_{y}(\mathrm{mrad} / \mathrm{s})$ & \pm 0.80 & \pm 0.85 & \pm 2.0 & \pm 0.94 \\
gyro $b_{z}(\mathrm{mrad} / \mathrm{s})$ & \pm 1.6 & \pm 0.62 & \pm 0.75 & \pm 0.58 \\
\hline
\end{tabular}

TABLE V

THE CALIBRATION PARAMETERS WITH $95 \%$ CONFIDENCE INTERVAL FOR THE GYROSCOPES, ACCELEROMETERS AND MAGNETOMETERS ESTIMATED FROM CALIBRATION MEASUREMENTS DONE ON AN INCLINED PLANE.

\begin{tabular}{r|rrr|r}
$\operatorname{diag}\left(\mathbf{D}_{\omega}\right)$ & \multicolumn{3}{|c|}{$\mathbf{D}_{\omega}^{-1} \mathbf{S}_{\omega}$} & $\mathbf{b}_{\omega}(\mathrm{mrad} / \mathrm{s})$ \\
\hline $0.9451 \pm 0.0007$ & $1.0000 \pm 0.0007$ & $0.0003 \pm 0.0011$ & $-0.0001 \pm 0.0020$ & $-0.95 \pm 0.76$ \\
$0.9435 \pm 0.0020$ & $-0.0010 \pm 0.0020$ & $1.0000 \pm 0.0020$ & $0.0023 \pm 0.0015$ & $2.5 \pm 0.81$ \\
$0.9820 \pm 0.0011$ & $0.0040 \pm 0.0020$ & $-0.0012 \pm 0.0015$ & $1.0000 \pm 0.0011$ & $1.7 \pm 0.74$ \\
\hline $\operatorname{diag}\left(\mathbf{D}_{a}\right)\left(\mu \mathrm{V} / \mathrm{m} / \mathrm{s}^{2}\right)$ & $\mathbf{D}_{a}^{-1} \mathbf{S}_{a}$ & $\mathbf{b}_{a}(\mathrm{mV})$ \\
\hline $6227.2 \pm 0.13$ & $0.9999 \pm 0.21 \mathrm{e}^{-4}$ & $0.0332 \pm 0.21 \mathrm{e}^{-4}$ & $0.0001 \pm 0.21 \mathrm{e}^{-4}$ & $1617.9 \pm 0.0008$ \\
$6267.4 \pm 0.11$ & $-0.0131 \pm 0.18 \mathrm{e}^{-4}$ & $0.9995 \pm 0.18 \mathrm{e}^{-4}$ & $-0.0024 \pm 0.19 \mathrm{e}^{-4}$ & $1622.6 \pm 0.0007$ \\
$6031.1 \pm 0.22$ & $-0.0021 \pm 0.37 \mathrm{e}^{-4}$ & $0.0015 \pm 0.37 \mathrm{e}^{-4}$ & $1.0000 \pm 0.36 \mathrm{e}^{-4}$ & $1608.1 \pm 0.0013$ \\
\hline $\left.\sin \left(\mathbf{D}_{B}\right)(\mathrm{V} / \mathrm{T})\right)$ & $\mathbf{D}_{B}^{-1} \mathbf{S}_{B}$ & \\
\hline $2651.9 \pm 0.047$ & $1.0000 \pm 0.18 \mathrm{e}^{-4}$ & $-0.0153 \pm 0.18 \mathrm{e}^{-4}$ & $0.0628 \pm 0.19 \mathrm{e}^{-4}$ & $2587.9 \pm 0.0015$ \\
$2270.4 \pm 0.047$ & $-0.0021 \pm 0.21 \mathrm{e}^{-4}$ & $0.9998 \pm 0.21 \mathrm{e}^{-4}$ & $-0.0052 \pm 0.22 \mathrm{e}^{-4}$ & $2538.6 \pm 0.0014$ \\
$2160.1 \pm 0.044$ & $0.0057 \pm 0.20 \mathrm{e}^{-4}$ & $0.0030 \pm 0.20 \mathrm{e}^{-4}$ & $0.9970 \pm 0.21 \mathrm{e}^{-4}$ & $2544.9 \pm 0.0013$ \\
\hline
\end{tabular}

There are small differences between the scale factors of magnetometers (diagonal elements of $\mathbf{D}_{B}$ ) between the calibration done on the horizontal plane (see Table II) and on the inclined plane. The calibration on the horizontal plane and on the inclined plane was done on the same place, but on a different day. In calculations, the magnitude of the magnetic field of the Earth was assumed to be the same $(\|\mathbf{B}\|=51000$ nT) in both calibrations. Since the difference in scale factors is of the same order between different measurement axes, this indicates that the magnitude of the magnetic field of the Earth may have slightly changed between the calibrations.

The confidence intervals of the calibration parameters on the inclined plane are smaller than the confidence intervals estimated on the horizontal plane. They suggest that more accurate calibration is obtained on the inclined plane. This is an effect from the fact that on inclined plane the acceleration measurements in different positions differed more from each other compared to the horizontal plane. The same holds also for the magnetic field measurements. Instead, the angular velocity measurements during the rotations are influenced by the user. On the inclined plane, the deviation of the rotations from a constant axis was greater than on the horizontal plane. Thus, calibration on an inclined plane with not-too-steady manual rotations is preferable.

In the calibration of the accelerometers the estimated angles $\alpha$ and $\beta$ were $15.0269 \pm 0.0007 \mathrm{deg}$. and $10.0926 \pm 0.0007$ deg. Thus, the method is also able to find the direction of the gravity. 


\section{Simulation results to show unbiasedness of the method}

The method is called unbiased, if the difference between true value and the expectation value of the parameter being estimated is zero. This can be tested with simulations. For this, the angular velocity, acceleration, and magnetic field data was created as explained in section V. The true calibration parameters are shown in the Table VI and the means of the estimated calibration parameters are shown in the Table VII with the means of $95 \%$ confidence interval.

For gyroscopes the differences between the true and the mean calibration parameters are within a thousandth. These results indicate that the calibration method of gyroscopes can be considered unbiased. In the simulations of the confidence intervals, it was noticed that the condition number of the matrix C, see Appendix B, was rather great in some noise realizations. This increases the confidence intervals.

For accelerometers the true and the mean of estimated calibration parameters are practically equal. The same holds also for magnetometers. Thus, the calibration method for accelerometers and magnetometers is unbiased. For accelerometers the means of the angles $\alpha$ and $\beta$ were $15.0000 \pm 0.0011$ deg. and $10.0000 \pm 0.0011 \mathrm{deg}$. and for magnetometers $15.0000 \pm 8.2 \mathrm{e}^{-4} \mathrm{deg}$. and $10.0000 \pm 8.1 \mathrm{e}^{-4} \mathrm{deg}$., which are equal to the true angle values $\alpha=15 \mathrm{deg}$. and $\beta=10 \mathrm{deg}$. in the simulation.

\section{CONCLUSION}

This paper presents an enhanced multi-position calibration method with a sensor error model of the biases, scale factors, misalignments, and cross-coupling errors of consumer-grade accelerometers, gyroscopes, and magnetometers. For magnetometers, hard iron and soft iron effect is also modelled. The results show that the gyroscopes can be calibrated successfully exploiting only known net rotations in multi-position calibration. In calibration of accelerometers and magnetometers the direction of the reference signals - the gravity and the magnetic field of the Earth - need be know only approximately in advance.

The estimated direction of the gravity, which can be easily measured with other method and thus compared to the estimated direction, was exploited to assess the success of the calibration of accelerometers. The $95 \%$ confidence intervals were calculated for the calibration parameters of the accelerometers, gyroscopes, and magnetometers to estimate the quality of the calibration results. In addition, simulations were done to show the unbiasedness of the method.

In practice, to calibrate the sensors with proposed method, no precise alignment of the sensors with respect to the reference signals and no additional measurement devices are needed: The calibration rotations can be done manually exploiting only a jig and a cube. Thus, the calibration can be done on the field. As a result, the method calibrates all the sensors at once to the same frame.

\section{APPENDIX A}

DERIVATION OF CONFIDENCE INTERVAL FOR CALIBRATION PARAMETERS OF ACCELEROMETERS AND MAGNETOMETERS

The calibration model for accelerometers and magnetometers is of the form

$$
\mathbf{h}(\tilde{\mathbf{x}})=\tilde{\mathbf{y}},
$$

where the actual measurements $\tilde{\mathbf{y}}=\mathbf{y}+\boldsymbol{\epsilon}$ contains the error free measurements $\mathbf{y}$ and the measurement errors $\boldsymbol{\epsilon}$. The elements of vector $\tilde{\mathbf{x}}=\mathbf{x}+\boldsymbol{\xi}$ are the calibration parameters, $\mathbf{x}$ contains the true calibration parameters and $\boldsymbol{\xi}$ is corresponding error due to $\epsilon$. The goal is to find the effect of $\epsilon$ on $\xi$.

To find the confidence interval for the estimated calibration parameters, the covariance matrix of $\boldsymbol{\xi}$, denoted by $\mathrm{V}(\boldsymbol{\xi})$, is first derived. The cost function of non-linear generalized least squares problem (13) is

$$
f(\mathbf{x})=(\mathbf{h}(\mathbf{x})-\mathbf{y})^{T} \mathrm{~V}(\tilde{\mathbf{y}})^{-1}(\mathbf{h}(\mathbf{x})-\mathbf{y}),
$$

where $\mathrm{V}(\tilde{\mathbf{y}})$ is the variance-covariance matrix of the measurements. Since variance-covariance matrix is a symmetric matrix, the derivative of the cost function is

$$
\frac{\partial f(\mathbf{x})}{\partial \mathbf{x}}=2 \mathbf{J}^{T} \mathrm{~V}(\tilde{\mathbf{y}})^{-1}(\mathbf{h}(\mathbf{x})-\mathbf{y}),
$$

where $\mathbf{J}=\mathbf{h}^{\prime}(\mathbf{x})$ is the Jacobian matrix. Since the space of the calibration parameters is unbounded, the minima of the function $f(\mathbf{x})$ occur at critical points $\mathbf{x}_{0}$, where $\frac{\partial f\left(\mathbf{x}_{0}\right)}{\partial \mathbf{x}}=\mathbf{0}$ holds.

For the actual measurements and the calibration parameters

$$
\mathbf{J}_{\tilde{\mathbf{x}}}^{T} \mathrm{~V}(\tilde{\mathbf{y}})^{-1}\left(\mathbf{h}\left(\mathbf{x}_{0}+\boldsymbol{\xi}\right)-(\mathbf{y}+\boldsymbol{\epsilon})\right)=\mathbf{0}
$$

holds, where $\mathbf{J}_{\tilde{\mathbf{x}}}=\mathbf{h}^{\prime}(\tilde{\mathbf{x}})$. Approximating the function $\mathbf{h}$ locally with Taylor sum $\mathbf{h}\left(\mathbf{x}_{0}+\boldsymbol{\xi}\right) \approx \mathbf{h}\left(\mathbf{x}_{0}\right)+\mathbf{J} \boldsymbol{\xi}$ and exploiting the fact that $\mathbf{J}_{\tilde{\mathbf{x}}}=\mathbf{J}$,

$$
\mathbf{J}^{T} \mathrm{~V}(\tilde{\mathbf{y}})^{-1}\left(\mathbf{h}\left(\mathbf{x}_{0}\right)-\mathbf{y}\right)+\mathbf{J}^{T} \mathrm{~V}(\tilde{\mathbf{y}})^{-1}(\mathbf{J} \boldsymbol{\xi}-\boldsymbol{\epsilon})=\mathbf{0}
$$

is obtained. Hence, the errors $\boldsymbol{\xi}$ and $\boldsymbol{\epsilon}$ satisfy

$$
\mathbf{J}^{T} \mathrm{~V}(\tilde{\mathbf{y}})^{-1} \mathbf{J} \boldsymbol{\xi}=\mathbf{J}^{T} \mathrm{~V}(\tilde{\mathbf{y}})^{-1} \boldsymbol{\epsilon}
$$

and subsequently

$$
\boldsymbol{\xi}=\left(\mathbf{J}^{T} \mathrm{~V}(\tilde{\mathbf{y}})^{-1} \mathbf{J}\right)^{-1} \mathbf{J}^{T} \mathrm{~V}(\tilde{\mathbf{y}})^{-1} \boldsymbol{\epsilon} .
$$

For the covariances of linear combinations

$$
\mathrm{V}(\boldsymbol{\xi})=\left(\mathbf{J}^{T} \mathrm{~V}(\tilde{\mathbf{y}})^{-1} \mathbf{J}\right)^{-1} \mathbf{J}^{T} \mathrm{~V}(\tilde{\mathbf{y}})^{-1} \mathrm{~V}(\boldsymbol{\epsilon})\left(\left(\mathbf{J}^{T} \mathrm{~V}(\tilde{\mathbf{y}})^{-1} \mathbf{J}\right)^{-1} \mathbf{J}^{T} \mathrm{~V}(\tilde{\mathbf{y}})^{-1}\right)^{T}
$$

holds [35], [36]. Exploiting the result $\mathrm{V}(\tilde{\mathbf{y}})=\mathrm{V}(\mathbf{y}+\boldsymbol{\epsilon})=$ $\mathrm{V}(\boldsymbol{\epsilon}), \mathrm{V}(\boldsymbol{\xi})$ reduces to

$$
\mathrm{V}(\boldsymbol{\xi})=\left(\mathbf{J}^{T} \mathrm{~V}(\boldsymbol{\epsilon})^{-1} \mathbf{J}\right)^{-1} .
$$

The covariance matrix of $\mathrm{V}(\boldsymbol{\epsilon})$ needs to be carefully accounted for, since each element of $\tilde{\mathbf{y}}$ is the mean of static sensor measurements - and it is, in fact, the maximum likelihood 
TABLE VI

THE CALIBRATION PARAMETERS EXPLOITED TO CREATE SIMULATIONS FOR THE GYROSCOPES, ACCELEROMETERS, AND MAGNETOMETERS.

\begin{tabular}{r|rrr|r}
$\operatorname{diag}\left(\mathbf{D}_{\omega}\right)$ & \multicolumn{3}{|c|}{$\mathbf{D}_{\omega}^{-1} \mathbf{S}_{\omega}$} & $\mathbf{b}_{\omega}(\mathrm{mrad} / \mathrm{s})$ \\
\hline 0.9581 & 1.0000 & 0.0003 & -0.0008 & -1.0000 \\
0.9539 & -0.0021 & 1.0000 & 0.0023 & 2.0000 \\
0.9765 & 0.0056 & -0.0015 & 1.0000 & 5.0000 \\
\hline $\operatorname{diag}\left(\mathbf{D}_{a}\right)\left(\mu \mathrm{V} / \mathrm{m} / \mathrm{s}^{2}\right)$ & $\mathbf{D}_{a}^{-1} \mathbf{S}_{a}$ & & $\mathbf{b}_{a}(\mathrm{mV})$ \\
\hline 5869.3 & 0.9964 & 0.0170 & 0.0852 & 1683.0 \\
6136.6 & 0.0652 & 0.9997 & -0.0163 & 1637.0 \\
6082.0 & -0.0493 & 0.0164 & 0.9965 & 1618.0 \\
\hline $\left.\operatorname{diag}\left(\mathbf{D}_{B}\right)(\mathrm{V} / \mathrm{T})\right)$ & \multicolumn{3}{|c|}{$\mathbf{D}_{B}^{-1} \mathbf{S}_{B}$} & \\
\hline 2246.4 & 1.0000 & 0.0089 & 0.0088 & $\mathbf{b}_{B}(\mathrm{mV})$ \\
2597.3 & 0.0010 & 1.0000 & -0.0007 & 2535.3 \\
2769.1 & -0.0031 & -0.0004 & 1.0000 & 2414.8 \\
\hline
\end{tabular}

TABLE VII

THE MEANS OF THE ESTIMATED CALIBRATION PARAMETERS FOR THE GYROSCOPES, ACCELEROMETERS, AND MAGNETOMETERS WITH $95 \%$ CONFIDENCE INTERVALS.

\begin{tabular}{r|rrr|r}
$\operatorname{diag}\left(\mathbf{D}_{\omega}\right)$ & \multicolumn{3}{|c|}{$\mathbf{D}_{\omega}^{-1} \mathbf{S}_{\omega}$} & $\mathbf{b}_{\omega}(\mathrm{mrad} / \mathrm{s})$ \\
\hline $0.9581 \pm 0.018$ & $1.0000 \pm 0.019$ & $0.0003 \pm 0.020$ & $-0.0009 \pm 0.018$ & $-0.9890 \pm 4.0$ \\
$0.9539 \pm 0.016$ & $-0.0021 \pm 0.016$ & $1.0000 \pm 0.017$ & $0.0023 \pm 0.014$ & $2.0011 \pm 3.8$ \\
$0.9765 \pm 0.015$ & $0.0056 \pm 0.011$ & $-0.0015 \pm 0.012$ & $1.0000 \pm 0.015$ & $5.0073 \pm 9.1$ \\
\hline $\operatorname{diag}\left(\mathbf{D}_{a}\right)\left(\mu \mathrm{V} / \mathrm{m} / \mathrm{s}^{2}\right)$ & $\mathbf{D}_{a}^{-1} \mathbf{S}_{a}$ & $\mathbf{b}_{a}(\mathrm{mV})$ \\
\hline $5869.3 \pm 0.19$ & $0.9964 \pm 0.31 \mathrm{e}^{-4}$ & $0.0170 \pm 0.31 \mathrm{e}^{-4}$ & $0.0852 \pm 0.31 \mathrm{e}^{-4}$ & $1683.0 \pm 0.0011$ \\
$6136.6 \pm 0.16$ & $0.0652 \pm 0.26 \mathrm{e}^{-4}$ & $0.9997 \pm 0.26 \mathrm{e}^{-4}$ & $-0.0163 \pm 0.26 \mathrm{e}^{-4}$ & $1637.0 \pm 0.0009$ \\
$6082.0 \pm 0.31$ & $-0.0493 \pm 0.51 \mathrm{e}^{-4}$ & $0.0164 \pm 0.51 \mathrm{e}^{-4}$ & $0.9965 \pm 0.51 \mathrm{e}^{-4}$ & $1618.0 \pm 0.0018$ \\
\hline $\left.\operatorname{siag}\left(\mathbf{D}_{B}\right)(\mathrm{V} / \mathrm{T})\right)$ & & $\mathbf{D}_{B}^{-1} \mathbf{S}_{B}$ & \\
\hline $2246.4 \pm 0.067$ & $1.0000 \pm 0.30 \mathrm{e}^{-4}$ & $0.0089 \pm 0.30 \mathrm{e}^{-4}$ & $0.0088 \pm 0.30 \mathrm{e}^{-4}$ & $2535.3 \pm 0.0020$ \\
$2597.3 \pm 0.065$ & $0.0010 \pm 0.25 \mathrm{e}^{-4}$ & $1.0000 \pm 0.25 \mathrm{e}^{-4}$ & $-0.0007 \pm 0.25 \mathrm{e}^{-4}$ & $2514.8 \pm 0.0019$ \\
$2769.2 \pm 0.058$ & $-0.0031 \pm 0.21 \mathrm{e}^{-4}$ & $-0.0004 \pm 0.21 \mathrm{e}^{-4}$ & $1.0000 \pm 0.21 \mathrm{e}^{-4}$ & $2487.6 \pm 0.0017$ \\
\hline
\end{tabular}

estimator in this case. If the covariance (in this case variance) of $n$ static sensor measurements $\mathbf{z}$ is $\Sigma$, the covariance of $\overline{\mathbf{z}}$ is

$$
\begin{aligned}
\mathrm{V}(\overline{\mathbf{z}}) & =\operatorname{cov}(\overline{\mathbf{z}}, \overline{\mathbf{z}})=\operatorname{cov}\left(\frac{1}{n} \sum_{i=1}^{n} z_{i}, \frac{1}{n} \sum_{j=1}^{n} z_{j}\right) \\
& =\frac{1}{n^{2}} \operatorname{cov}\left(\sum_{i=1}^{n} z_{i}, \sum_{j=1}^{n} z_{j}\right)=\frac{1}{n^{2}} n \Sigma=\frac{1}{n} \Sigma
\end{aligned}
$$

since $\operatorname{cov}\left(z_{i}, z_{i}\right)=\Sigma$ and $\operatorname{cov}\left(z_{i}, z_{j}\right)=0$ for $i \neq j$ assuming that the measurements are independent. That is, the covariance of one set of the mean measurements is the covariance of the measurements multiplied by $1 / n$. In each position three sets of dependent measurements are gathered. Accordingly, the covariance matrix of three sets of the mean measurements is the covariance matrix of three sets of measurements $\boldsymbol{\Sigma}_{i} \in \mathbb{R}^{3 \times 3}$ multiplied by $1 / n$. The measurements concerning different positions are independent. Thus, the off-diagonal blocks of $\mathrm{V}(\boldsymbol{\epsilon})$ are zero matrices and $\mathrm{V}(\boldsymbol{\epsilon})=\frac{1}{n} \operatorname{diag}\left(\left[\boldsymbol{\Sigma}_{1}, \boldsymbol{\Sigma}_{2}, \ldots, \boldsymbol{\Sigma}_{m}\right]\right)$.

The diagonal elements of the matrix $\mathrm{V}(\boldsymbol{\xi})$ are the estimated variances of the error in the elements of the calibration parameters $\mathbf{S}_{i}$ and $\mathbf{b}_{i}$. The error variances of the elements of $\mathbf{D}_{i}$ were calculated exploiting the fact that the square of a standard normal distribution is a chi-squared distribution. The error variances of the elements of $\mathbf{D}_{i}^{-1} \mathbf{S}_{i}$ were approximated by assuming that the errors of the diagonal elements of $\mathbf{D}$ are zero. Thus, for the elements of $\mathbf{D}_{i}^{-1} \mathbf{S}_{i}$ only the order of the error variance is given. In general, the square root of the variance is the standard deviation $\sigma$ and $1.96 \sigma$ constitutes the $95 \%$ confidence interval in normal distribution.

\section{APPENDIX B}

\section{DERIVATION OF CONFIDENCE INTERVAL FOR CALIBRATION} PARAMETERS OF GYROSCOPES

The cost function $f(\mathbf{x}, \mathbf{y})$ of the Tikhonov regularization problem (9) can be given as

$$
f(\mathbf{x}, \mathbf{y})=\mathbf{r}^{T}(\mathbf{x}, \mathbf{y}) \mathbf{r}(\mathbf{x}, \mathbf{y})+\lambda(\mathbf{G} \mathbf{x}-\mathbf{y})^{T}(\mathbf{G} \mathbf{x}-\mathbf{y}),
$$

where $\mathbf{x}$ is unknown (containing the quaternions and calibration parameters) and the elements of $\mathbf{y}$ are the measurements (angular velocities and reference positions). By exploiting the cost function, the goal is to estimate the effect of the error in the measurements on the calibration parameters. For this, the derivative of the cost function is

$$
\frac{\partial f(\mathbf{x}, \mathbf{y})}{\partial \mathbf{x}}=2 \mathbf{J}_{x}^{T} \mathbf{r}(\mathbf{x}, \mathbf{y})+2 \lambda \mathbf{G}^{T}(\mathbf{G} \mathbf{x}-\mathbf{y})
$$

where $\mathbf{J}_{x}^{T}=\frac{\partial \mathbf{r}^{T}(\mathbf{x}, \mathbf{y})}{\partial \mathbf{x}}$ is the Jacobian matrix. Since the space of the calibration parameters is unbounded, the minima of the function $f(\mathbf{x}, \mathbf{y})$ occur at critical points $\mathbf{x}_{0}$, where $\frac{\partial f\left(\mathbf{x}_{0}, \mathbf{y}\right)}{\partial \mathbf{x}}=$ 0 holds.

The error $\delta \mathbf{y}$ in measurements will cause an error $\delta \mathbf{x}$ to the solution $\mathbf{x}_{0}$. To find the relation between $\delta \mathbf{y}$ and $\delta \mathbf{x},(25)$ is approximated with a 1st order Taylor polynomial in the neighborhood of the critical point

$$
\begin{array}{r}
\frac{\partial f\left(\mathbf{x}_{0}+\delta \mathbf{x}, \mathbf{y}+\delta \mathbf{y}\right)}{\partial \mathbf{x}} \approx \\
\frac{\partial f\left(\mathbf{x}_{0}, \mathbf{y}\right)}{\partial \mathbf{x}}+\frac{\partial^{2} f\left(\mathbf{x}_{0}, \mathbf{y}\right)}{\partial \mathbf{x}^{2}} \delta \mathbf{x}+\frac{\partial^{2} f\left(\mathbf{x}_{0}, \mathbf{y}\right)}{\partial \mathbf{y} \partial \mathbf{x}} \delta \mathbf{y}=\mathbf{0}
\end{array}
$$


where $\frac{\partial^{2} f\left(\mathbf{x}_{0}, \mathbf{y}\right)}{\partial \mathbf{x}^{2}}=2\left(\frac{\partial \mathbf{J}_{x}^{T}}{\partial \mathbf{x}} \mathbf{r}\left(\mathbf{x}_{0}, \mathbf{y}\right)+\mathbf{J}_{x}^{T} \mathbf{J}_{x}+\lambda \mathbf{G}^{T} \mathbf{G}\right)=\mathbf{C}$ and $\frac{\partial^{2} f\left(\mathbf{x}_{0}, \mathbf{y}\right)}{\partial \mathbf{y} \partial \mathbf{x}}=2\left(\frac{\partial \mathbf{J}_{x}^{T}}{\partial \mathbf{y}} \mathbf{r}\left(\mathbf{x}_{0}, \mathbf{y}\right)+\mathbf{J}_{x}^{T} \mathbf{J}_{y}-\lambda \mathbf{G}^{T}\right)=\mathbf{D}$. With this notation, (26) reduces to

$$
\mathbf{C} \delta \mathbf{x}=-\mathbf{D} \delta \mathbf{y} .
$$

Exploiting the identity (21), the covariance matrix of $\delta \mathbf{x}$ is

$$
\mathrm{V}(\delta \mathbf{x})=\mathbf{C}^{-1} \mathbf{D V}(\delta \mathbf{y}) \mathbf{D}^{T}\left(\mathbf{C}^{T}\right)^{-1}
$$

since $\mathbf{C}$ is a square matrix.

The diagonal elements of the matrix $\mathrm{V}(\delta \mathbf{x})$ are the variances of the errors of the quaternions and the variances of the error in the elements of the calibration parameters $\mathbf{S}_{\omega}^{-1}$ and $\mathbf{b}_{\omega}$. The measurement covariance matrix $\mathrm{V}(\delta \mathbf{y})$ is chosen to be a diagonal matrix, whose diagonal elements are the variances of the errors in the angular velocity measurements and the reference position. The variances of the errors in the angular velocities can be estimated from static sensor measurements. The errors of the reference positions are assumed to be zero.

To find the error variances of the elements of $\mathbf{D}_{\omega}$ and $\mathbf{D}_{i}^{-1} \mathbf{S}_{i}$, the error variances of the elements $\mathbf{S}_{\omega}$ were first estimated exploiting a 1st order Taylor polynomial and assuming that the errors in $\mathbf{S}_{\omega}^{-1}$ do not correlate. Thereafter the error variances of the elements of $\mathbf{D}_{\omega}$ and $\mathbf{D}_{\omega}^{-1} \mathbf{S}_{\omega}$ were calculated as explained in Appendix A.

\section{REFERENCES}

[1] P. D. Groves, Principles of GNSS, Inertial and Multisensor Integrated Navigation Systems, 2nd ed. Artech House, 2013.

[2] D. H. Titterton and J. L. Weston, Strapdown Inertial Navigation Technology, 2nd ed. London and Reston, VA: IET and AIAA, 2004.

[3] A. B. Chatfield, Fundamentals of High Accuracy Inertial Navigation, Washington, DC, USA: AIAA, 1997.

[4] Y. Li, X. Niu, Q. Zhang, H. Zhang, and C Shi, "An in situ hand calibration method using a pseudo-observation scheme for low-end inertial measurement units," Meas. Sci. Technol., vol. 23, no. 10, p. 105104, Aug. 2012.

[5] W. T. Fong, S. K. Ong, and A. Y. C. Nee, "Methods for in-field user calibration of an inertial measurement unit without external equipment," Meas. Sci. Technol., vol. 19, no. 8, p. 085202, Jul. 2008.

[6] Z. F. Syed, P. Aggarwal, C. Goodall, X. Niu, and N. El-Sheimy, "A new multi-position calibration method for MEMS inertial navigation systems," Meas. Sci. Technol., vol. 18, no. 7, pp. 1897-1907, May 2007.

[7] T. Nieminen, J. Kangas, S. Suuriniemi, and L. Kettunen, ”An enhanced multi-position calibration method for consumer-grade inertial measurement units applied and tested," Meas. Sci. Technol., vol. 21, no. 10, p. 105204, Aug. 2010

[8] I. Skog and P. Handel, "Calibration of MEMS inertial measurement unit," in Proc. 17th IMEKO World Congr. Metrol. Sustain. Develop., Sep. 2006, pp. 1722.

[9] M. Hwangbo and T. Kanade, "Factorization-based calibration method for MEMS inertial measurement unit," IEEE Int. Conf. on Robotics and Automation pp. 1306-1311, 2008.

[10] T. Forsberg, N. Grip, and N. Sabourova, "Non-iterative calibration for accelerometers with three non-orthogonal axes, reliable measurement setups and simple supplementary equipment," Meas. Sci. Technol., vol. 24, no. 3, p. 035002, Feb. 2012.

[11] J. C. Lötters, J. Schipper, P. H. Veltink, W. Olthuis, and P. Bergveld, "Procedure for in-use calibration of triaxial accelerometers in medical applications," Sens. Actuat. A Phys., vol. 68, nos. 1-3, pp. 221-228, Jun. 1998.

[12] M. Glueck, D. Oshinubi, P. Schopp, and Y. Manoli, "Real-time autocalibration of MEMS accelerometers," IEEE Trans. Instrum. Meas., vol. 63, no. 1, pp. 96-105, Jan. 2014.

[13] S. Won and F. Golnaraghi, "A triaxial accelerometer calibration method using a mathematical model," IEEE Trans. Instrum. Meas., vol. 59, no. 8, pp. 2144-2153, Aug. 2010.
[14] X. Li and Z. Li, "Vector-aided in-field calibration method for low-end MEMS gyros in attitude and heading reference systems," IEEE Trans. Instrum. Meas., vol. 63, no. 11, pp. 2675-2681, Nov. 2014.

[15] Y. Li and J. Georgy, X. Niu, Q. Li, and N. El-Sheimy, "Autonomous calibration of MEMS gyros in consumer portable devices," IEEE $S$. Journal, vol. 15, no.71, pp. 4062-4072, Jul. 2015.

[16] X. Li and Z. Li, "A new calibration method for tri-axial field sensors in strap-down navigation systems," Meas. Sci. Technol., vol. 23, no. 10 p. 105105 , Sep. 2012.

[17] J. M. G. Merayo, P. Brauer, F. Primdahl, J. R. Petersen, and O. V. Nielsen, "Scalar calibration of vector magnetometers," Meas. Sci. Technol., vol. 11, no. 2, pp. 120-132, 2000.

[18] H. U. Auster, K. H. Fornacon, E. Georgescu, K. H. Glassmeier, and U. Motschmann, "Calibration of flux-gate magnetometers using relative motion," Meas. Sci. Technol., vol. 13, no. 7, pp. 1124-1131, Jun. 2002.

[19] J. F. Vasconcelos, G. Elkaim, C. Silvestre, P. Oliveira, and B. Cardeira, "Geometric approach to strapdown magnetometer calibration in sensor frame," IEEE Trans. Aerosp. Electron. Syst., vol. 47, no. 2, pp. 12931306, Apr. 2011.

[20] V. Renaudin, H. Afzal, and G. Lachapelle, "Complete triaxis magnetometer calibration in the magnetic domain," J. Sensors, vol. 2010, pp. $1-10,2010$.

[21] J. Metge, R. Mégret, A. Giremus, Y. Berthoumieu, and T. Décamps, "Calibration of an inertial-magnetic measurement unit without external equipment, in the presence of dynamic magnetic disturbances," Meas. Sci. Technol., vol. 25, no. 12, p. 125106, Oct. 2014.

[22] G. Secer and B. Barshan, "Improvements in deterministic error modeling and calibration of inertial sensors and magnetometers," Sens. Actuat. A Phys., vol. 247, pp. 522-538, Aug. 2016.

[23] Z. Zhang, "Two-step calibration methods for miniature inertial and magnetic sensor units," IEEE Trans. Industrial Electron., vol. 62, no. 6, pp. 3714-3723, Jun. 2015.

[24] WolframAlpha [Online]. Available: http://www.wolframalpha.com/ widgets/view.jsp?id=d34e8683df527e3555153d979bcda9cf

[25] National Centres for Environmental Information, address: 151 Patton Ave, Asheville, NC 28801, North Carolina, U.S.A. https://www.ngdc. noaa.gov/geomag/magfield.shtml

[26] A. Iserles, A First Course in the Numerical Analysis of Differential Equations, Cambridge University Press, 1996.

[27] P. C. Hansen, Rank-Deficient and Discrete Ill-Posed Problems: Numerical Aspects of Linear Inversion, Philadelphia, PA: SIAM, 1998.

[28] D. P. Bertsekas, Nonlinear Programming, 2nd ed. Nashua, NH: Athena Scientific, 1999

[29] STMicroelectronics LPY4150AL. [Online]. Available: https://media. digikey.com/pdf/Data Sheets/ST Microelectronics PDFS/LPY4150AL. pdf

[30] Å. Björck, Numerical Methods for Least Squares Problems, Philadelphia, PA: SIAM, 1996.

[31] Analog Devices ADXL326. [Online]. Available: http://www.analog.com/ media/en/technical-documentation/data-sheets/ADXL326.pdf

[32] Honeywell HMC1043. [Online]. Available: https://media.digikey.com/ pdf/Data Sheets/Honeywell PDFs/HMC1043.pdf

[33] O. Särkkä, T. Nieminen, S. Suuriniemi, and L. Kettunen, "Augmented inertial measurements for analysis of javelin throwing mechanics," Sports Eng., vol. 19, no. 4, pp. 219-227, Dec. 2016.

[34] Nurmijärvi Geophysical Observatory, address: Observatoriontie 125, 05100 Röykkä, Finland

[35] B. W. Lindgren, Statistical Theory, 3rd ed. New York: MacMillan, 1976.

[36] R. C. Aster, B. Borchers, C. H. Thurber, Parameter estimation and inverse problems, 2nd ed. Academic Press, 2013.

Olli Särkkä received a M.Sc. degree in electrical engineering from the Tampere University of Technology, Tampere, Finland, in 2009. He is currently a doctoral student at the Tampere University of Technology.

Tuukka Nieminen received a D.Sc. degree in electrical engineering from the Tampere University of Technology, Tampere, Finland, in 2013. He is currently working in the private sector. 
Saku Suuriniemi received a D.Sc. degree in electrical engineering from the Tampere University of Technology, Tampere, Finland, in 2004. He is currently working in the private sector.

Lauri Kettunen received a D.Sc. degree in electrical engineering from the Tampere University of Technology, Tampere, Finland, in 1992. He is currently a Research Professor at the University of Jyvaskyla, Finland. 